\title{
A Study on the Innovative Design Method of Rural Micro- landscape — Taking Edible Fungi as an Example
}

\author{
Bin-jie $\mathrm{LUO}^{1 *}$ \\ ${ }^{1}$ TED Academy Kookmin University Seoul, Korea 02707
}

\begin{abstract}
Innovation is not only the motive force of scientific and technological development, but also an important strategy to advance steadily in the process of beautiful rural construction and rural revitalization and development, and also the core driving force of rural landscape development. Based on this, taking the edible fungi of rural plants as an example, this paper expounds the edible fungi which play an important role in the process of rural development, analyzes the formation, characteristics and functions of the microlandscape of edible fungi, and draws lessons from the traditional Chinese landscape techniques, from the four dimensions of main scene and matching scene, real scene and virtual scene, penetrating and leaking scene, screen view and borrowing scene, to discusses the innovative design method of rural micro-landscape, and provide innovative design ideas for rural landscape development.
\end{abstract}

\section{Introduction}

In the context of rural development, edible fungi companies and farmers have begun to change their business ideas, and new creative bonsais based on planting edible fungi have gradually emerged, and they have been exported to Italy, Netherlands, Denmark and other countries to open up the development of edible fungi micro-landscapes. However, the earliest fungus as a bonsai model is Ganoderma lucidum in China. To better reflect its artistic value, the fungus originally used for edible and medicinal purposes is processed by baking and drying, shaping, and antiseptic. Long-term preservation of fungus ornamental [1], but the safety of dried Ganoderma bonsai is not good, and creativity is limited, so instead, attention is paid to the microlandscape with live edible fungus bonsai. However, due to its short development time, a complete landscape design system has not yet been formed. So taking edible fungi as an example, discuss the design methods of rural micro-landscapes, give full play to the higher ornamental value of edible fungi, continue the unique natural form of edible fungi, and further promote the development of rural landscapes.

\section{Research method}

Scope

\subsection{The role of edible fungus micro-landscape}

The Romans believed that edible fungus was "God's food." The Greeks once believed that eating edible fungus could improve the warrior's combat effectiveness, and edible fungus was called the "life panacea" in China. Edible mushroom bonsai, as a new type of horticulture that combines viewing and eating, solding to the domestic and overseas markets, creating considerable economic value for the innovative development of edible mushrooms.

Edible fungi are cultivated in courtyards or rooms, and can absorb oxygen and release carbon dioxide during the growth process, which can improve the environment; children, teenagers or gardening enthusiasts can observe and summarize the growth process and growth patterns of plants by cultivating fungi plants to form observations. Picking and eating at maturity can improve the quality of life such as nutrition and health; planting edible fungi in miniature bonsai for morphological landscaping, and improve the artistic accomplishment of the subject of appreciation. Through the organic combination of edible fungi and natural landscape, it reflects the vitality and market competitiveness of edible fungi ${ }^{[2]}$, and fully reflects the comprehensive value that is respectable, rewarding and edible.

\subsection{Features of edible fungus micro-landscape}

Edible fungus is a common fungus plant in nature and can be eaten by humans. The edible fungus microlandscape is rich in fungus resources, diverse in variety, rich in nutrition, short in shape and other factors. It has distinctive features such as rich shape, edible and health care value, and strong ornamental. It is extremely convenient to artificially cultivate and can be used as a home, office, etc., for landscaping.

First, the nutritious edible value. The edible fungi resources are extremely rich in China, with more than

\footnotetext{
* Corresponding author e-mail: anglelbj@126.com
} 
300 varieties. Because edible fungus is rich in trace elements, vitamins, minerals and other nutrients that are beneficial to the human body, it also contains natural organic germanium, polymer polysaccharides and other biologically active substances. Zhang Jinxia, a researcher at the Chinese Academy of Agricultural Sciences, once said: "Edible fungus is a functional health food, both nutritious and delicious." [3]. For these reasons, it can be used as home grown ornamental and edible.

Second, the unique shape and convenient production. The edible mushroom micro-landscape is influenced by the famous landscape paintings and poems of the Tang Dynasty. It has traditional Chinese classical gardens and landscape bonsai with "three-dimensional landscape paintings". In terms of composition and modeling, it is similar to Chinese landscape painting, and contains the philosophy of "one sand, one world, one leaf and one bodhi". It has the advantages of easy acquisition of materials, short forming time, simple production, etc. It is a natural and relatively friendly art piece ${ }^{[4]}$.

Third, the rich ornamental value. Different types of edible fungi have different shapes and graceful postures, such as mushroom-shaped, umbrella-shaped, net-shaped, and filamentous. For example, the appearance of oyster mushroom and Xiuzhen mushroom are like petals; the appearance of hericium is like monkey's head ${ }^{[5]}$. The various forms are rich in levels, including big and small, top and bottom, and front and back patchy, sparse and dense, thick and thin, and there are colors in gradation and contrast; caps, stipes, cells, gills, etc. Many color elements, such as white, yellow, brown, pink, and gray. The landscaping of the fungus entity has strong plasticity and good ornamental value, which can create abundant of edible fungi micro-landscape effects for viewers.

\subsection{Method}

By literatures review, learned about the related research of edible fungi and landscape design from the network resources, such as Research on the Design and Development of Edible Fungus Creative Landscape, and so on. Net database and electronic library, read landscape design books, and intensively read the related literature materials of edible fungi theme and landscape design methods in recent years, such as Planning and design of Youxiyangzhong edible fungi theme sightseeing park. Then, forms clear research questions gradually.

Datas induction, based on the collection and induction of network, books, pictures and other data, summarizes the development direction of edible fungi, analyzes that the formation of edible fungi is composed of natural growth and artificial creation, and summarizes the characteristics of unique micro-landscape, ample nutritions, ornamental, edible and health care value.

Case depth analyses method, according to the graphic datas of edible fungi minibonsai, makes comprehensive observations and analyses. From the perspective of micro-landscape, this paper makes a deep study on the design method, then draws a conclusion.

\section{Discussion}

\subsection{Micro-landscape Innovation Design of Edible Fungi}

Landscaping is human's unremitting pursuit of "spatial beauties" and the aesthetic cognition of aesthetic subjects to aesthetic objects. As early as the Paleolithic age of primitive society, humans began to portray, record and create beautiful things on stone walls. Therefore, edible fungi are used as the main object of human consumption and aesthetic creation to construct a better life at home. In order to express the specific spatial landscape and spatial characteristics in the garden landscape design, make the best of environmental conditions, rationally use landscaping elements, and create a good-effect edible fungus micro landscape, it is necessary to consider the landscape effect of edible fungi in a specific container from multiple perspectives. And the micro-space changes, from the following four dimensions, construct a landscaping technique based on the edible fungus micro landscape.

\subsection{Main scene and secondary scene}

Taking edible fungi as the main scenery is the focal point and core of the scenery and the main element of the scenery. The main body can be emphasized by shifting the center of gravity of the composition. The main scenery is highlighted with the supporting scenery, so that the main scenery becomes the central view of the whole micro-landscape, making the contrast between the main scenery interesting ${ }^{[6]}$, and enhancing the artistic charm of the edible fungus micro-landscape. The differencet, point of view and angle produces microsecond changes, such as looking up, looking down (Fig 1); In composition, the central main scene or the main axis focus and the four sides of the landscape method; the main scene color area is higher than the auxiliary scene color. In order to highlight the spectacular effect of edible fungi, the main scene needs foreground and background as auxiliary scenery, and the density of bacteria needs to comprehensively study the difference effect between the forms of microspace, so as to perfect the multi-angle display of microspace form and create high quality conditions. Therefore, it is necessary to increase the depth of landscape and enrich the landscape level according to the change of color and form.

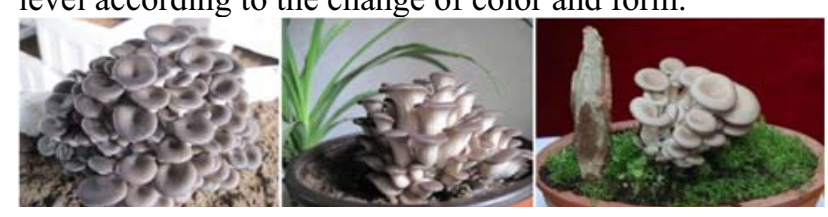

Fig.1 Micro-space morphology of edible fungi micro-landscape

\subsection{Real scene and virtual scene}

The real scene and the virtual scene of the edible fungus micro-landscape mean that there is reality in the virtual 
and the virtual in reality. The virtual and the reality are coexisted and blended, and through the contrast between the virtual and the reality, the virtual and the reality are penetrated to create a rich visual landscape. The artistic form, posture, light, etc., constituting the edible fungus micro-landscape are virtual scenery. Artistic form is to express regular or irregular through art and image language, and construct aesthetic activities before the participants or experiencers of aesthetic subjects. On the basis of protecting and continuing the natural form of edible fungi, the artistic form of mycelial plants is fully demonstrated (Fig 2). Take advantage of the contrast between the virtual and the real, expand the space, increase the poetic and artistic flavor, and form a natural landscape painting ${ }^{[7]}$.

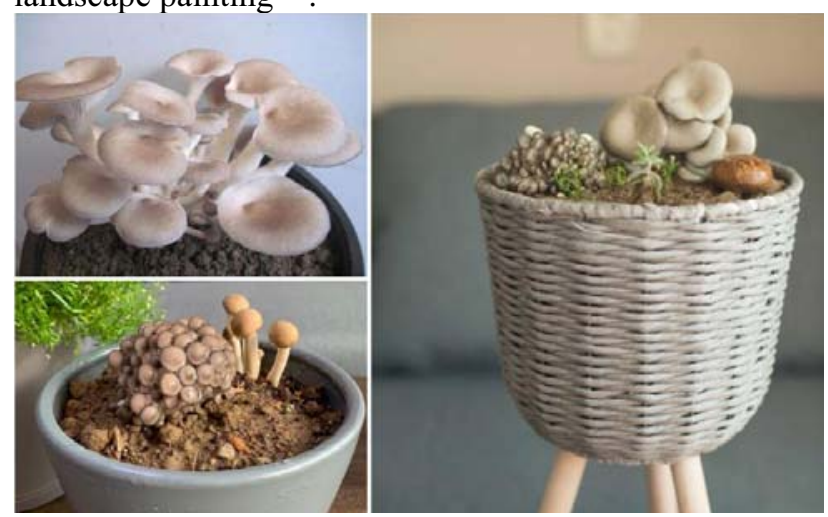

Fig.2 Artistic form of edible fungus micro-landscape

In order to create a feeling space similar to music and dance, present a kind of agile and circular life state ${ }^{[8]}$. Take the Cap Fungus bonsai as an example, the form of Cap Fungus from a top view (Fig 2) is naturally radial, densely distributed from the center point to multiple directions; from a head-up point of view, the scattered bacteria will highlight the scenery. The characteristics of "leak, transparent, thin and thick" show unique artistic image and artistic connotation, inspire people's artistic identification with natural life with edible fungi as the main body, and embody the virtual landscape created by people's unique soul for life art.

Take planting techniques and natural forms as real scenes. The natural form of edible fungus is the form that edible fungi grows freely according to natural laws during the life movement process such as seeding and growth of fungi, primordium differentiation, mycelial growth, and maturation of fungi. For example, the surface of the cap of Coprinus comatus is like a thorny mushroom (Fig 3). Therefore, the real scene is the great creation of edible fungus, which bred a rich natural language form, which is reflected in the spatial form of different edible fungi as the main scene.

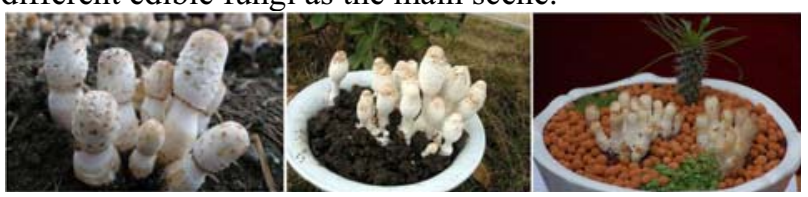

Fig.3 The natural form of edible fungus micro-landscape

\subsection{Perspective scene and leakage scene}

Perspective can be expressed as unobstructed "transparency" between the front and rear scenery, and the scenery can completely "see through" into a new place. The perspective and framed scenery have the same effect. They can be "permeated" through the window frames, porches, door frames and other structures of the building. The outer contour of the structure is used as the edge of the composition to frame the surrounding scenery and be potted with edible mushrooms. Through leaky window lattices, fences, walls and other geometric decorative buildings, consciously place the edible mushroom bonsai in front of the framed building, and introduce the scenery outside the scene or the garden in a looming way, such as pomegranate, bamboo, winter plum, old tree, etc.

It can also be used to leak scenes through dense patterns, regular or irregular graphics naturally formed on the surface of the fungus, and the combination of "points, lines, and surfaces" with concavities and convexities. The uniformity and diversity of texture forms constitute an image "body" of virtual and real. It embodies the beauty of the "qi" and "rhyme" of the leaked scene. The texture shape is affected by the texture material, color and form, etc., then finally forms different bumpy texture forms on the texture surface. For example, the morel shape resembles a sheep tripe with a pointed top and a concave honeycomb shape on the surface. The composition of "line and net" and the formation of "face and body" are scattered with dense; the outline form of bamboo fungus "line and net" like a princess wearing a gauze skirt, dark green mushroom cap. And the "body" of the snow-white cylindrical stem, and the "net"-like stem similar to the white snake skin, form a "body net" form, highlighting the beautiful texture of the bacterial body (Fig 4).

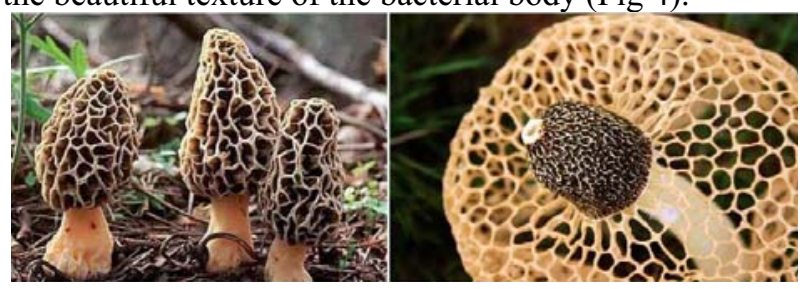

Fig.4 Surface texture of edible fungi

\subsection{Shielding scene and borrowing scene}

Shielding and borrowing scenes are referred to as screen borrowed. Ji Cheng put forward in "Yuanye": "The good is to accept it, the vulgar is to screen it", absorbing the good scenery, which means borrowing the scene; blocking or hiding other undesirable scenery, means the shielding scene. Borrowing scenery is a landscaping technique in traditional Chinese gardens, which can make up for the influence of small spatial scale. Because of the limited space and area of potted edible mushrooms, to enhance the depth of the edible mushroom microlandscape, the bonsai was "borrowed" and superimposed with the edible mushroom bonsai scene to make a roundabout landscape. 
There are many ways to borrow scenery, such as borrowing from neighbors, nearby scenery of potted plants, background or texture of water features, countertops, window sills, and other decorative objects; borrowing from a distance inside or outside the building walls, background etc.; cleverly, through different viewing angles, cleverly integrate the water surface reflection, blue sky and white clouds, lights and sunlight with edible mushroom potted plants to form a rich landscape effect; borrow from different time, according to the changes of the seasons, or the shape and color of the seasonal plants, to create diversified texture forms; based on meteorological landscape, meteorological changes, through sunrise, dusk, sunset, rain and snow, etc., also present an instant dynamic landscape, and form a micro-spaces with distinct layers.

\section{Conclusion}

This study is focused on the edible fungus microlandscape. Because resources are extremely rich, with different shapes among varieties. The development time of edible fungus bonsai as a micro-landscape is relatively short. To develop gardening bonsai into a landscape that the public is willing to accept. It needs to be based on healthy nutrition, according to the characteristics of the edible fungus micro-landscape, combined with traditional garden landscape. The edible fungus micro-landscape should take nature first, learn from the art of landscaping in classical gardens, imitate nature and the artistic beauty of transcending nature, not stick to fixed landscaping patterns, and take full advantage of the various characteristics and rich shapes of edible fungi to reflect. The beauty of the virtual and the reality of different landscaping techniques forms the beauty of the overall micro-landscape, which maximizes the condensed landscape of "within a few minutes, far away".

\section{References}

1. Xie Chunqin, Yu Baofeng, Li Xinlan, Shi Jun, Yang Hetong. Analysis on the production technology of edible fungus art ornamental products $[\mathrm{J}]$. Edible fungus, 2011, 33(01): 59-60+67.

2. Feng Zhen. Research on the Design and Development of Edible Fungus Creative Landscape[J]. Edible Fungus in China, 2019, 38(01): 113-116.

3. Lin Jia, Li Changfa, Tang Lihua. New technology of Ganoderma bonsai modeling[J]. Edible fungus, 2015, 37(04): 47-49.

4. Mao Lihui, Wen Congfa, Ding Huaqiao, Zou Qingcheng, Tian Danqing. Landscape value of bryophytes[J]. China Wild Plant Resources, 2020, 39(07): 30-32+38.

5. Wang Jiamei. Planning and design of Youxiyangzhong edible fungi theme sightseeing park[D]. Fujian Agriculture and Forestry University, 2018.
6. Wu Man. On the application of traditional gardening techniques in modern landscape design[J]. Art Critic, 2017(04): 170-173.

7. Lei Fang, Hu Xijun, Li Mengting, Jiang Zhe, Cheng Shishi. Analysis of the landscaping form of "natural celestial phenomena"[J]. Northern Horticulture, 2015(03): 88-92.

8. Peng Xiuyin. The role of urban sculpture as a public art in the construction of urban space aesthetics[J]. Architecture and Culture, 2015(10):19. 